Research Article

\title{
Positive Observer Design for Positive Delayed Markovian Jump Systems
}

\author{
Guoliang Wang \\ School of Information and Control Engineering, Liaoning Shihua University, Fushun, Liaoning 113001, China \\ Correspondence should be addressed to Guoliang Wang; gliangwang@aliyun.com
}

Received 20 April 2016; Revised 20 June 2016; Accepted 29 June 2016

Academic Editor: Rigoberto Medina

Copyright (C) 2016 Guoliang Wang. This is an open access article distributed under the Creative Commons Attribution License, which permits unrestricted use, distribution, and reproduction in any medium, provided the original work is properly cited.

\begin{abstract}
The positive observer design problem is considered for a class of positive delayed Markovian jump systems (PDMJSs). Firstly, a necessary and sufficient condition is established to check the positivity of delayed Markovian jump systems (DMJSs). Then, necessary and sufficient conditions for PDMJSs being asymptotically mean stable are established to be independent of time delay. Based on the proposed results, sufficient conditions for the existence of the desired positive observer gains including modeindependent case are provided. Additionally, more general cases that transition rate matrix (TRM) is partially unknown or uncertain are considered, respectively. Finally, a numerical example is used to demonstrate the effectiveness of the proposed methods.
\end{abstract}

\section{Introduction}

Markovian jump system (MJS) is a kind of hybrid systems and usually has two mechanisms simultaneously. The first one is the time-evolving mechanism and related to the state vector. The second one referring to the system mode is the event-driven mechanism and driven by the Markov process. During the past years, a lot of attention has been paid to various kinds of MJSs; see, for example, [1-12]. As we know, time delay is usually encountered in practically dynamical systems, such as in chemical systems, manufacturing systems, biological systems, networked control systems (NCSs), and telecommunication and economic systems. It is said that the presence of time delay is often a big source of instability and poor performance. During the past decades, many results on DMJSs have been reported; see, for example, [13-19] and the references therein.

Positive systems have formed a new branch and play an important role in system theory and application. It is a kind of systems whose state and output are nonnegative values for any given nonnegative initial states and inputs. Such systems are usually found in many areas including population models, economics, ecology, and communication [20-23], whose variables with negative values have no physical meaning. Over the past several decades, a great number of results on various topics such as positive realization [24], reachability $[25,26]$, and positive stabilization [27-30] have emerged. When there are time delays, some results were reported in [31-34]. By investigating such references, it is seen that the underlying system is a deterministic system without jumping parameters, where many other systems cannot be described. In this sense, it is said that the results about deterministic systems have limited application scope. Thus, it is meaningful to study systems with random jumps. If positive systems have their parameters and structures changed abruptly, it is necessary and natural to describe them as positive Markovian jump systems (PMJSs). Up to the present, it is seen that there are very few results to report this kind of systems. Very recently, the stochastic stability of continuous-time PMJSs was considered in [35]. In this reference, it is seen that there is no time delay, and only analysis problems were considered. By investigating this reference and the process of this paper, it is said that the existence of time delay will make the analysis and synthesis of PMJSs very difficult. As for discretetime PMJSs, the $l_{1}$-gain performance and positive filter design were studied in [36], where the transition probabilities may be partially unknown or have interval uncertainties. However, the case of general TRMs in continuous-time MJSs is quite different from the case of general transition probability matrices (TPMs) in discrete-time MJSs. So, the 
corresponding problems for continuous-time PMJSs with general TRMs should be revisited. Up to now, it is found that the effects of time delay and general TRMs in continuoustime system analysis and synthesis have not been mentioned so much. More importantly, in such two references, it is priorly assumed that the underlying system is positive. In other words, how to check its positivity is not referred to in these references, especially when time delay is included. On the other hand, due to technical or economical constraints in most practical applications, it is not easy or expensive to get all the state variables. In this case, it is of great interest to design observer to estimate state variables. The observer design problems for deterministic systems were considered in [37-40], while similar problems for MJSs were investigated in [41-44]. By investigating these references, it is said that the observer design techniques developed for generally dynamical systems may not be suitable for positive systems. The reason is that there is often a positive constraint on the observer design for positive systems [45-48]. Similarly, from the above references, it is known that such references are all without jump and time delay. However, based on the explanations, it is said that such general factors to be considered for PMJSs play important roles in positive analysis and synthesis, which should not be simply combined. To the best of our knowledge, the positive observer design problem of PMJSs has not yet been fully investigated and still remains challenging. In order to spell out the applications of the considered problems, without loss of generality, only a practical example is referred to here. From [49], it is seen that a single-link robot arm could be modeled to be an MJS with two modes, whose payload and moment of inertia have different modes. By applying some controller and assuming time delay in the above model, one could have a practical system naturally, which is an PMJS to be considered here. Based on these facts and illustrations, it is claimed that the studies of such general factors in positive systems have theoretical and practical senses. All the observations motivate the current research.

In this paper, we will study the positive observer design problem for continuous-time PDMJSs. The main contributions of this paper are summarized as follows: (1) necessary and sufficient condition for the positivity of DMJSs is presented; (2) necessary and sufficient conditions for checking its asymptotically mean stability are developed, which can also be reduced to check the asymptotic stability of a deterministic system without time delay; (3) based on these results, sufficient conditions for designing observers including mode-independent observer are established, which are convenient to be solved by using the standard software; (4) the given results are further extended to two general cases that TRM is partially unknown and has admissible uncertainties, respectively.

Notation. $\mathbb{R}^{n}$ denotes the $n$ dimensional Euclidean space, and $\mathbb{R}^{m \times n}$ is the set of all $m \times n$ real matrices. $\mathbb{Z}^{+}$is the set of positive integer. $\mathbb{R}_{+}^{m \times n}$ is the set of all $m \times n$ real matrices with nonnegative entries, and the set of $n \times n$ Metzler matrices is denoted by $\mathbb{M}_{n}$. A real matrix or vector $M$ is called positive (resp., strictly positive) and denoted by $M \succeq 0$ (resp., $M>0$ ), if all its components are nonnegative (resp., strictly positive). $\mathscr{E}[\cdot]$ means the mathematical expectation of $[\cdot]$. vec $[\cdot]$ is an operation and consists of taking the columns of a given matrix from left to right and stacks them one above the other. $x \mathrm{~V}$ $y$ denotes the maximum of $x$ and $y$. In symmetric block matrices, we use “*” as an ellipsis for the terms induced by symmetry, $\operatorname{diag}\{\cdots\}$ for a block-diagonal matrix, and $M^{\star} \triangleq$ $M+M^{T}$.

\section{Positive Delayed Markovian Jump Systems}

Consider a class of delayed Markovian jump systems described as

$$
\begin{aligned}
& \dot{x}(t)=A\left(\eta_{t}\right) x(t)+A_{d}\left(\eta_{t}\right) x(t-d)+B\left(\eta_{t}\right) u(t), \\
& y(t)=C\left(\eta_{t}\right) x(t)+D\left(\eta_{t}\right) u(t),
\end{aligned}
$$

where $x(t) \in \mathbb{R}^{n}$ is the system state, $u(t) \in \mathbb{R}^{m}$ is the input, and $y(t) \in \mathbb{R}^{p}$ is the output. $A\left(\eta_{t}\right) \in \mathbb{R}^{n \times n}, A_{d}\left(\eta_{t}\right) \in \mathbb{R}^{n \times n}$, $B\left(\eta_{t}\right) \in \mathbb{R}^{n \times m}, C\left(\eta_{t}\right) \in \mathbb{R}^{p \times n}$, and $D\left(\eta_{t}\right) \in \mathbb{R}^{p \times m}$ are known matrices. Operation mode $\left\{\eta_{t}, t \geq 0\right\}$ is a homogeneous stationary ergodic Markov process taking values in a finite set $\mathbb{S}=\{1,2, \ldots, N\}$ with TRM $\Pi=\left(\pi_{i j}\right) \in \mathbb{R}^{N \times N}$ :

$$
\operatorname{Pr}\left\{\eta_{t+h}=j \mid \eta_{t}=i\right\}= \begin{cases}\pi_{i j} h+o(h) & i \neq j \\ 1+\pi_{i i} h+o(h) & i=j\end{cases}
$$

where $h>0, \lim _{h \rightarrow 0^{+}}(o(h) / h)=0$, and $\pi_{i j} \geq 0$, if $i \neq j$, $\pi_{i i}=-\sum_{j \in \mathbb{S}} \pi_{i j}$. The initial condition of system (1) is defined as

$$
x(t)=x_{0}(t), \quad \forall t \in[-d, 0],
$$

where $x_{0}(t)$ is a given vector function and is continuous and $d \geq 0$ is the time delay.

Definition 1. System (1) is said to be positive, if and only if $x(t) \in \mathbb{R}_{+}^{n}, y(t) \in \mathbb{R}_{+}^{p}$, for any $x_{0}(t) \in \mathbb{R}_{+}^{n}$ and $u(t) \in \mathbb{R}_{+}^{m}$, $\forall t \geq 0, \forall \eta_{0} \in \mathbb{S}$.

Theorem 2. System (1) is positive if and only if $A_{i} \in \mathbb{M}_{n}, A_{d i} \in$ $\mathbb{R}_{+}^{n \times n}, B_{i} \in \mathbb{R}_{+}^{n \times m}, C_{i} \in \mathbb{R}_{+}^{p \times n}$, and $D_{i} \in \mathbb{R}_{+}^{p \times m}, \forall i \in \mathbb{S}$.

Proof.

Necessity. Define the stopping time instants $0=t_{0}<t_{1}<$ $t_{2}<\cdots<t_{k}<\cdots$ satisfying $\eta_{t_{0}}=i_{0}, \eta_{t_{1}}=i_{1}, \eta_{t_{2}}=$ $i_{2}, \ldots, \eta_{t_{k}}=i_{k}$; we have $\eta_{t}=\eta_{t_{0}}=i_{0}, t \in\left[t_{0}, t_{1}\right), \eta_{t}=\eta_{t_{1}} \stackrel{2}{=} i_{1}$, $t \in\left[t_{1}, t_{2}\right), \ldots, \eta_{t}=\eta_{t_{k}}=i_{k}, t \in\left[t_{k}, t_{k+1}\right)$. Without loss of generality, it is assumed that $t_{1} \leq d$. Let system (1) with $x_{0}(t)=0, \forall t \in[-d, 0]$, and $u(t)=0, \forall t \geq 0$; we have

$$
\dot{x}(t)=A_{i_{0}} x(t), \quad t \in\left[0, t_{1}\right) .
$$

It is known that $x(t) \in \mathbb{R}_{+}^{n}$, only if $A_{i_{0}} \in \mathbb{M}_{n}$. Assume $x_{0}(-d)=\mathbb{e}_{i}, i=1,2, \ldots, n$, where $\mathbb{e}_{i}$ is the $i$ th column of identical matrix $I_{n}$ and $x_{0}(0)=0$; when $t=0$, one has $\dot{x}(0)=A_{d i_{0}} \mathbb{e}_{\ell}=A_{d i_{0}}^{\ell} \in \mathbb{R}_{+}^{n}$, where $A_{d i_{0}}^{\ell}$ is the $\ell$ th column 
of $A_{d i_{0}}$. Thus, we obtain $A_{d i_{0}} \in \mathbb{R}_{+}^{n}$. Let system (1) be with $x_{0}(t)=0, \forall t \in[-d, 0]$, and $u(t)=0, \forall t \geq 0$; we have $\dot{x}(0)=B_{i_{0}} u(0) \in \mathbb{R}_{+}^{n}$. Then, $B_{i_{0}} \in \mathbb{R}_{+}^{n \times m}$, since $u(0) \in \mathbb{R}_{+}^{m}$ is arbitrary. The necessities of $C_{i_{0}} \in \mathbb{R}_{+}^{p \times n}$ and $D_{i_{0}} \in \mathbb{R}_{+}^{p \times m}$ can be proved similarly. Because of Markov process $\left\{\eta_{t}, t \geq 0\right\}$ being an ergodic Markov process, any mode can take place and is also selected as an initial mode. Then, we have that $\eta_{t_{0}}=i_{0}$ discussed above can be any mode in the finite set $\mathbb{S}$. For case $t_{1}>d$, the corresponding time range can be selected as $[0, d]$. Then, by the similar method, we can get the above results too.

Sufficiency. When $t \in[0, d]$, without loss of generality, there are some stopping time instants such that $0=t_{0}<t_{1}<t_{2}<$ $\cdots<t_{k}<t_{k+1}$ and $t_{k} \leq d<t_{k+1}$. The occupying modes are $\eta_{t_{0}}=i_{0}, \eta_{t_{1}}=i_{1}, \ldots, \eta_{t_{k}}=i_{k}$, and the original delay rang $[0, d]$ is divided as $[0, d]=\left[t_{0}, t_{1}\right) \cup\left[t_{1}, t_{2}\right) \cup\left[t_{2}, t_{3}\right) \cup \cdots \cup\left[t_{k}, d\right]$. Then, as for $t \in\left[t_{0}, t_{1}\right)$, the solution to system (1) is

$$
\begin{aligned}
x(t)= & e^{A_{i_{0}} t} x_{0}+\int_{0}^{t} e^{A_{i_{0}}(t-s)} A_{d i_{0}} x_{0}(s-d) d s \\
& +\int_{0}^{t} e^{A_{i_{0}}(t-s)} B_{i_{0}} u(s) d s .
\end{aligned}
$$

Because of $A_{i_{0}} \in \mathbb{M}_{0}, A_{d i_{0}} \in \mathbb{R}_{+}^{n \times n}$, and $B_{i_{0}} \in \mathbb{R}_{+}^{n \times m}$, it is concluded that $x(t) \in \mathbb{R}_{+}^{n}, \forall t \in\left[t_{0}, t_{1}\right)$, if $x_{0}(t) \in \mathbb{R}_{+}^{n}$ and $u(t) \in \mathbb{R}_{+}^{m}$. Moreover, we have $y(t) \in \mathbb{R}_{+}^{p}$. Similarly, when $t \in\left[t_{1}, t_{2}\right)$, we obtain that

$$
\begin{aligned}
x(t)= & e^{A_{i_{1}}\left(t-t_{1}\right)} x\left(t_{1}\right)+\int_{t_{1}}^{t} e^{A_{i_{1}}(t-s)} A_{d i_{1}} x(s-d) d s \\
& +\int_{t_{1}}^{t} e^{A_{i_{1}}(t-s)} B_{i_{1}} u(s) d s,
\end{aligned}
$$

where $x\left(t_{1}\right)=x\left(t_{1}^{-}\right) \in \mathbb{R}_{+}^{n}$. By the given conditions in this theorem and $u(t) \in \mathbb{R}_{+}^{m^{+}}$, it is obvious that $x(t) \in \mathbb{R}_{+}^{n}$, $t \in\left[t_{1}, t_{2}\right)$. Similarly, we have $x(t) \in \mathbb{R}_{+}^{n}, t \in[0, d]$. By the step method, one has $x(t) \in \mathbb{R}_{+}^{n}$ on the intervals $[d, 2 d],[2 d, 3 d], \ldots$. Finally, we have $x(t) \in \mathbb{R}_{+}^{n}$ on $[0,+\infty)$. This completes the proof.

Remark 3. In [45-48], the positivity of the considered systems was assumed to be satisfied beforehand. In other words, in these references, no results are available to check its positivity. Based on Theorem 2, one could check an MJS with time delay positive or not by only testing some system matrices.

\section{Stability Analysis}

Definition 4. The positive system in (1) is asymptotically mean stable if and only if the solution to system (1) for $u(t)=$ 0 satisfies

$$
\lim _{t \rightarrow \infty} \mathscr{E}[x(t)]=0
$$

for any $x_{0}(t) \in \mathbb{R}_{+}^{n}, \forall t \in[-d, 0], \forall \eta\left(t_{0}\right) \in \mathbb{S}$.

Theorem 5. The following statements are equivalent: (i) The positive system in (1) is asymptotically mean stable.

(ii) There exists a strictly positive vector $(S P V) \lambda \in \mathbb{R}_{+}^{n N}$ such that

$$
\widehat{A} \lambda \prec 0,
$$

where matrix $\widehat{A} \in \mathbb{R}^{n N \times n N}$ is defined as

$$
\widehat{A}=\left[\begin{array}{cccc}
\bar{A}_{1}+\pi_{11} I_{n} & \pi_{21} I_{n} & \ldots & \pi_{N 1} I_{n} \\
\pi_{12} I_{n} & \bar{A}_{2}+\pi_{22} I_{n} & \ldots & \pi_{N 2} I_{n} \\
\vdots & \vdots & \ddots & \vdots \\
\pi_{1 N} I_{n} & \pi_{2 N} I_{n} & \ldots & \bar{A}_{N}+\pi_{N N} I_{n}
\end{array}\right] .
$$

It is equivalent to

$$
\bar{A}_{i} \lambda_{i}+\sum_{j=1}^{n} \pi_{j i} \lambda_{j} \prec 0
$$

where $\bar{A}_{i}=A_{i}+A_{d i}$, and $S P V \lambda_{i} \in \mathbb{R}_{+}^{n}, \forall i \in \mathbb{S}$, is to be determined.

(iii) Matrix $\widehat{A}$ is Hurwitz.

(iv) The positive system without time delay

$$
\dot{x}(t)=\widehat{A} x(t)
$$

is asymptotically stable. That is, a positive defined matrix $P \in \mathbb{R}^{n N \times n N}$ satisfies

$$
\widehat{A}^{T} P+P \widehat{A}<0 .
$$

(v) The positive transform system

$$
\dot{x}(t)=\widehat{A}^{T} x(t)
$$

is asymptotically stable. It is equivalent to matrix $\widehat{A}^{T} \epsilon$ $\mathbb{R}^{n N \times n N}$ being Hurwitz, which is also equivalent to the fact that there exists a positive defined matrix $P \in$ $\mathbb{R}^{n N \times n N}$ such that

$$
\widehat{A} P+P \widehat{A}^{T}<0 .
$$

(vi) There exists an $S P V \lambda \in \mathbb{R}_{+}^{n N}$ such that

$$
\lambda^{T} \widehat{A} \prec 0
$$

which is rewritten to be an $S P V \lambda_{i} \in \mathbb{R}_{+}^{n}$ satisfying

$$
\lambda_{i}^{T} \bar{A}_{i}+\sum_{j=1}^{N} \pi_{i j} \lambda_{j}^{T} \prec 0
$$

or

$$
\bar{A}_{i}^{T} \lambda_{i}+\sum_{j=1}^{N} \pi_{i j} \lambda_{j} \prec 0 .
$$


Proof. Let $\mathscr{E}[x(t)]=q(t)$, where $q(t)=\sum_{i=1}^{N} q_{i}(t)$. Here, $q_{i}(t)$ is defined as $q_{i}(t)=\mathscr{E}\left[x(t) \mathbf{1}_{\{\alpha(t)=i\}}(\omega)\right]$, where the indicator function is given by

$$
\mathbf{1}_{\{\alpha(t)=i\}}(\omega)= \begin{cases}1, & \text { if } \alpha(t)=i \in \mathbb{S} \\ 0, & \text { otherwise. }\end{cases}
$$

It is concluded that

$$
\begin{aligned}
d q_{i}( & \\
= & \mathscr{E}\left[(d x(t)) \mathbf{1}_{\{\alpha(t)=i\}}(\omega)+x(t) d\left(\mathbf{1}_{\{\alpha(t)=i\}}(\omega)\right)\right] \\
= & A_{i} \mathscr{E}\left[(d x(t)) \mathbf{1}_{\{\alpha(t)=i\}}(\omega)\right] \\
& +A_{d i} \mathscr{E}\left[(d x(t-d)) \mathbf{1}_{\{\alpha(t)=i\}}(\omega)\right] \\
& +\sum_{j=1}^{N} \pi_{j i} \mathscr{E}\left[(d x(t)) \mathbf{1}_{\{\alpha(t)=j\}}(\omega)\right] .
\end{aligned}
$$

From the results $[13,15,50]$, it is known that $\left\{x_{t}, \eta_{t}\right\}_{t \geq 0}$ is also a Markov process, where $x_{t}(s)=x(t+s),-d \leq s \leq t$. Then, based on (19) and considering the definition of $q_{i}(t)$, we have

$$
\dot{q}_{i}(t)=A_{i} q_{i}(t)+A_{d i} q_{i}(t-d)+\sum_{j=1}^{N} \pi_{j i} q_{i}(t) .
$$

Let $v_{q}(t)=\operatorname{vec}\left[q_{i}(t)\right]$; one has

$$
\dot{v}_{q}(t)=\widetilde{A} v_{q}(t)+\widetilde{A}_{d} v_{q}(t-d),
$$

where

$$
\begin{gathered}
\widetilde{A}=\left[\begin{array}{cccc}
A_{1}+\pi_{11} I_{n} & \pi_{21} I_{n} & \cdots & \pi_{N 1} I_{n} \\
\pi_{12} I_{n} & A_{2}+\pi_{22} I_{n} & \cdots & \pi_{N 2} I_{n} \\
\vdots & \vdots & \ddots & \vdots \\
\pi_{1 N} I_{n} & \pi_{2 N} I_{n} & \cdots & A_{N}+\pi_{N N} I_{n}
\end{array}\right], \\
\widetilde{A}_{d}=\left[\begin{array}{cccc}
A_{d 1} & & & \\
& A_{d 2} & & \\
& & \ddots & \\
& & A_{d N}
\end{array}\right] .
\end{gathered}
$$

Then, the stability of positive system (1) is equivalent to positive system (21).

(i) $\Leftrightarrow$ (ii) Necessity. From system (21), it is obtained that

$$
v_{q}(\infty)-v_{q}(0)-\widetilde{A}_{d} \int_{-d}^{0} v_{q}(s) d s=\widehat{A} \int_{0}^{+\infty} v_{q}(s) d s,
$$

where $\widehat{A}=\widetilde{A}+\widetilde{A}_{d}$. Because of positive system (21) asymptotically stable, it is seen that when $v_{q}(\infty)=0, v_{q}(0)+$ $\widetilde{A}_{d} \int_{-d}^{0} v_{q}(s) d s>0$ and $\int_{0}^{+\infty} v_{q}(s) d s>0$. Thus, we conclude

$$
\widehat{A} \int_{0}^{+\infty} v_{q}(s) d s \prec 0 .
$$

Let $\lambda \triangleq \int_{0}^{+\infty} v_{q}(s) d s \in \mathbb{R}_{+}^{n N}$; we obtain (8).
Sufficiency. It is obvious that the stability of system (1) is equivalent to the stability of system (21) which is also a positive system with $v_{q}(t) \in \mathbb{R}_{+}^{n}$. Choose a Lyapunov function for system (21) defined by

$$
V(x)=v_{q}^{T}(t) \lambda+\int_{t-d}^{t} v_{q}^{T}(s) \widetilde{A}_{d} \lambda d s
$$

which is positive for any nonzero $x(t) \in \mathbb{R}_{+}^{n}$. Then, one gets

$$
\begin{aligned}
\dot{V}(x)= & \dot{v}_{q}^{T}(t) \lambda+v_{q}^{T}(t) \widetilde{A}_{d} \lambda-v_{q}^{T}(t-d) \widetilde{A}_{d} \lambda \\
= & v_{q}^{T}(t) \widetilde{A} \lambda+v_{q}^{T}(t-d) \widetilde{A}_{d} \lambda+v_{q}^{T}(t) \widetilde{A}_{d} \lambda \\
& -v_{q}^{T}(t-d) \widetilde{A}_{d} \lambda=v_{q}^{T}(t)\left(\widetilde{A}+\widetilde{A}_{d}\right) \lambda<0
\end{aligned}
$$

since $\widehat{A} \lambda \prec 0$ and nonzero $v_{q}(t) \in \mathbb{R}_{+}^{n}$. Let $\lambda=$ $\left[\begin{array}{llll}\lambda_{1}^{T} & \lambda_{2}^{T} & \cdots & \lambda_{N}^{T}\end{array}\right]^{T}$ and, substituting it into (8), it is concluded that conditions (8) and (10) are equivalent.

(ii) $\Leftrightarrow$ (iii) As we know, by [51], the Metzler matrix $\widehat{A}$ is Hurwitz if and only if there exists an SPV $\lambda \in \mathbb{R}_{+}^{n N}$ such that $\widehat{A} \lambda<0$.

(iii) $\Leftrightarrow$ (iv) It is known that matrix $\widehat{A}$ being Hurwitz can be equivalently described by the asymptotic stability of system (11). Moreover, its asymptotic stability is equal to LMI (12) holding.

(iv) $\Leftrightarrow$ (v) By the dual theory, one concludes that the asymptotic stabilities of system (11) and its dual system are equivalent.

(v) $\Leftrightarrow$ (vi) It is obvious that, for any SPV $\lambda \in \mathbb{R}_{+}^{n}$, it is equivalent to $\lambda^{T}>0$. Based on this fact, one gets this conclusion right. That is because matrix $\widehat{A}$ being Hurwitz is equivalent to matrix $\widehat{A}^{T}$ being Hurwitz. The next proof on the equivalence can be obtained by the proof of equivalence of (ii) $\Leftrightarrow$ (iv) similarly.

This completes the proof.

Remark 6. Here, several kinds of equivalent conditions are proposed with different forms; the choice of form to be chosen should depend on the concrete situations. First, some conditions such as (8), (10), and (15) are very conveniently used to check its asymptotically mean stability. Second, some other forms, such as (16) or (17), are very suitably applied to general cases that TRM is partially unknown or uncertain. Such conditions will make the synthesis problems of PDMJSs with or without general TRMs in this paper easily. Third, by fully considering the positivity of MJSs, some conditions are obtained by constructing a linear copositive stochastic Lyapunov function. Compared with the results without positivity constraint, the computational complexity for checking the stability of PMJSs can be reduced. Finally, it is seen that PDMJS (1) is asymptotically mean stable if and only if deterministically positive system (11) is asymptotically stable. In other words, it is said that the checking of stability of PDMJS (1) is reduced to check whether the corresponding positive system without time delay is stable or not.

From Theorem 5, it is seen that corresponding TRM $\Pi$ is exactly known and will have the application limited. When it 
is more general, such as being partially unknown or uncertain, how to obtain the similar results will become necessary. Firstly, we consider the case of $\Pi$ partially unknown. Without loss of generality, a partly unknown $\Pi$ may be expressed as

$$
\Pi=\left[\begin{array}{cccc}
\pi_{11} & \pi_{12} & ? & ? \\
\pi_{21} & ? & ? & \pi_{24} \\
? & ? & \pi_{33} & ? \\
\pi_{41} & \pi_{42} & \pi_{43} & \pi_{44}
\end{array}\right],
$$

where "?" represents the unknown element. Then for any $i \in$ $\mathbb{S}$, defining $\mathbb{S}^{i}=\mathbb{S}_{k}^{i}+\overline{\mathbb{S}}_{k}^{i}$ such that

$$
\begin{aligned}
& \mathbb{S}_{k}^{i}=\left\{j: \pi_{i j} \text { is known }\right\}, \\
& \overline{\mathbb{S}}_{k}^{i}=\left\{j: \pi_{i j} \text { is unknown }\right\}
\end{aligned}
$$

with $\sigma=\min _{i \in \overline{\mathbb{S}}_{k}^{i}}\left\{\pi_{i i}\right\}$ known, which are further described as

$$
\begin{aligned}
& \mathbb{S}_{k}^{i}=\left\{k_{1}^{i}, \ldots, k_{m}^{i}\right\}, \\
& \overline{\mathbb{S}}_{k}^{i}=\left\{\bar{k}_{1}^{i}, \ldots, \bar{k}_{N-m}^{i}\right\},
\end{aligned}
$$

where $k_{j}^{i} \in \mathbb{Z}^{+}$is the column index of the $j$ th known element in the $i$ th row of $\Pi$, and the column index of the $(N-j)$ th unknown element in the $i$ th row of $\Pi$ is represented as $\bar{k}_{N-j}^{i} \epsilon$ $\mathbb{Z}^{+}$. For this general case, based on Theorem 5, we have the following result.

Theorem 7. The positive system in (1) with partially unknown TRM is asymptotically mean stable if there are SPVs $\lambda_{i} \in \mathbb{R}_{+}^{n}$ and $w_{i} \in \mathbb{R}_{+}^{n}$, such that

$$
\begin{gathered}
\lambda_{i}^{T} \bar{A}_{i}+\sum_{j \neq i, j \in \mathbb{S}_{k}^{i}} \pi_{i j}\left(\lambda_{j}^{T}-\lambda_{i}^{T}-w_{i}^{T}\right)-\pi_{i i} w_{i}^{T} \prec 0, \\
\quad i \in \mathbb{S}_{k}^{i}, \\
\lambda_{i}^{T} \bar{A}_{i}+\sum_{j \neq i, j \in \mathbb{S}_{k}^{i}} \pi_{i j}\left(\lambda_{j}^{T}-\lambda_{i}^{T}-w_{i}^{T}\right)-\sigma w_{i}^{T} \prec 0, \\
\quad i \in \overline{\mathbb{S}}_{k}^{i}, \\
\lambda_{j}^{T}-\lambda_{i}^{T}-w_{i}^{T} \preceq 0, \\
j \neq i \in \overline{\mathbb{S}}_{k}^{i} .
\end{gathered}
$$

Proof. Based on Theorem 5, it is obtained that positive system (1) is asymptotically mean stable if condition (16) holds, which is equivalent to

$$
\begin{gathered}
\lambda_{i}^{T} \bar{A}_{i}+\sum_{j \neq i, j \in \mathbb{S}_{k}^{i}} \pi_{i j}\left(\lambda_{j}^{T}-\lambda_{i}^{T}-w_{i}^{T}\right)-\pi_{i i} w_{i}^{T} \\
+\sum_{j \neq i, j \in \overline{\mathbb{S}}_{k}^{i}} \pi_{i j}\left(\lambda_{j}^{T}-\lambda_{i}^{T}-w_{i}^{T}\right) \prec 0,
\end{gathered}
$$

where $w_{i} \in \mathbb{R}_{+}^{n}$. It is further guaranteed by

$$
\begin{array}{r}
\lambda_{i}^{T} \bar{A}_{i}+\sum_{j \neq i, j \in \mathbb{S}_{k}^{i}} \pi_{i j}\left(\lambda_{j}^{T}-\lambda_{i}^{T}-w_{i}^{T}\right)-\pi_{i i} w_{i}^{T} \prec 0, \\
\sum_{j \neq i, j \in \overline{\mathbb{S}}_{k}^{i}} \pi_{i j}\left(\lambda_{j}^{T}-\lambda_{i}^{T}-w_{i}^{T}\right) \preceq 0 .
\end{array}
$$

If $\eta_{t}=i \in \mathbb{S}$ is known such that $i \in \mathbb{S}_{k}^{i}$, we have (34) equal to (30). On the contrary, if $i \in \overline{\mathbb{S}}_{k}^{i}$ is unknown, it is obtained that (34) can be guaranteed by (31). As for (35), because of $\pi_{i j} \geq 0, j \neq i$ and $j \in \overline{\mathbb{S}}_{k}^{i}$, one gets that (32) implies (35). This completes the proof.

When $\Pi$ is uncertain, it is assumed to have an admissible uncertainty

$$
\Pi=\widetilde{\Pi}+\Delta \widetilde{\Pi}
$$

in which $\widetilde{\Pi} \triangleq\left(\widetilde{\pi}_{i j}\right)$ is an estimation of $\Pi$ and $\Delta \widetilde{\Pi} \triangleq\left(\Delta \widetilde{\pi}_{i j}\right)$ with $\Delta \tilde{\pi}_{i j} \triangleq \pi_{i j}-\tilde{\pi}_{i j}$ and $\alpha_{i j} \triangleq \tilde{\pi}_{i j}-\epsilon_{i j}$ is the estimated error with property (2) and $\Delta \tilde{\pi}_{i j}, j \neq i$, takes any value in $\left[-\epsilon_{i j}, \epsilon_{i j}\right]$. Moreover, it is obtained that $\left|\Delta \tilde{\pi}_{i i}\right| \leq-\epsilon_{i i}$ with $\epsilon_{i i} \triangleq-\sum_{j=1, j \neq i}^{N} \epsilon_{i j}$. Then, we have the following result.

Theorem 8. The positive system in (1) is robustly asymptotically mean stable on all admissible uncertainty if there are SPVs $\lambda_{i} \in \mathbb{R}_{+}^{n}$ and $w_{i} \in \mathbb{R}_{+}^{n}, \forall i \in \mathbb{S}$, satisfying

$$
\begin{aligned}
& \lambda_{i}^{T} \bar{A}_{i}+\sum_{j \neq i} \alpha_{i j}\left(\lambda_{j}^{T}-\lambda_{i}^{T}\right)+2 \sum_{j \neq i} \epsilon_{i j} w_{i}^{T} \prec 0, \\
& \lambda_{j}^{T}-\lambda_{i}^{T}-w_{i}^{T} \preceq 0, \quad j \neq i .
\end{aligned}
$$

Proof. Based on Theorem 5, it is obtained that system (1) is asymptotically mean stable if there exists an SPV $\lambda_{i} \in \mathbb{R}_{+}^{n}$ such that

$$
\lambda_{i}^{T} \bar{A}_{i}+\sum_{j=1}^{N} \pi_{i j} \lambda_{j}^{T} \prec 0 .
$$

Consider condition (36); it is equivalent that there exists an SPV $\lambda_{i} \in \mathbb{R}_{+}^{n}$ satisfying

$$
\lambda_{i}^{T} \bar{A}_{i}+\sum_{j \neq i}^{N} \tilde{\pi}_{i j}\left(\lambda_{j}^{T}-\lambda_{i}^{T}\right)+\sum_{j \neq i}^{N} \Delta \tilde{\pi}_{i j}\left(\lambda_{j}^{T}-\lambda_{i}^{T}\right) \prec 0
$$

which is also equivalent to

$$
\begin{aligned}
\lambda_{i}^{T} \bar{A}_{i} & +\sum_{j \neq i} \alpha_{i j}\left(\lambda_{j}^{T}-\lambda_{i}^{T}\right) \\
& +\sum_{j \neq i}\left(\Delta \pi_{i j}+\epsilon_{i j}\right)\left(\lambda_{j}^{T}-\lambda_{i}^{T}-w_{i}^{T}\right) \\
& +\sum_{j \neq i}\left(\Delta \tilde{\pi}_{i j}+\epsilon_{i j}\right) w_{i}^{T} \prec 0,
\end{aligned}
$$


where $w_{i} \in \mathbb{R}_{+}^{n}$. Because of $\Delta \pi_{i j}+\epsilon_{i j} \geq 0$ and $w_{i}^{T} \geq 0$, we have the above inequality guaranteed by

$$
\lambda_{i}^{T} \bar{A}_{i}+\sum_{j \neq i} \alpha_{i j}\left(\lambda_{j}^{T}-\lambda_{i}^{T}\right)+\sum_{j \neq i}\left(\Delta \tilde{\pi}_{i j}+\epsilon_{i j}\right) w_{i}^{T} \prec 0
$$

and (38). Based on the definition (36), it is concluded that (42) is ensured by (37). This completes the proof.

\section{Positive Observer Design}

For positive Markovian jump system (1), consider the following observer described by

$$
\begin{aligned}
\dot{\hat{x}}(t)= & A\left(\eta_{t}\right) \hat{x}(t)+A_{d}\left(\eta_{t}\right) \hat{x}(t-d) \\
& +L\left(\eta_{t}\right)(\hat{y}(t)-y(t)), \\
\hat{y}(t)= & C\left(\eta_{t}\right) \hat{x}(t),
\end{aligned}
$$

where $\widehat{x}(t)$ is the estimate of $x(t), \widehat{y}(t)$ is the output, and $L\left(\eta_{t}\right)$ is the gain of the designed observer. Let $e(t)=\widehat{x}(t)-x(t)$; if $e(t) \in \mathbb{R}_{+}^{n}$, because of $x(t) \in \mathbb{R}_{+}^{n}$, we have $\hat{x}(t) \in \mathbb{R}_{+}^{n}$. Thus, we obtain

$$
\begin{aligned}
\dot{e}(t)= & A\left(\eta_{t}\right) e(t)+A_{d}\left(\eta_{t}\right) e(t-d) \\
& +L\left(\eta_{t}\right) C\left(\eta_{t}\right) e(t) \\
= & \left(A\left(\eta_{t}\right)+L\left(\eta_{t}\right) C\left(\eta_{t}\right)\right) e(t) \\
& +A_{d}\left(\eta_{t}\right) e(t-d) .
\end{aligned}
$$

By the above proposed theorems, we have the following results.

Theorem 9. Consider positive system (1); there exists positive observer (43) such that error system (44) is asymptotically mean stable if there exist matrices $P_{i}=\left(p_{\mu \nu}^{i}\right)>0$ and $Y_{i} \in$ $\mathbb{R}_{+}^{n \times p}, i \in \mathbb{S}$, satisfying

$$
\begin{gathered}
p_{\mu \nu}^{i} \leq 0, \quad \forall \mu \neq \nu \in\{1,2, \ldots, n\}, \\
{\left[\begin{array}{cccc}
\bar{\Omega}_{1} & \Phi_{12} & \cdots & \Phi_{1 N} \\
* & \bar{\Omega}_{2} & \cdots & \Phi_{2 N} \\
\vdots & \vdots & \ddots & \vdots \\
* & \cdots & \cdots & \bar{\Omega}_{N}
\end{array}\right]<0}
\end{gathered}
$$

where

$$
\begin{aligned}
\bar{\Omega}_{i} & =\left(\bar{A}_{i}^{T} P_{i}+C_{i}^{T} Y_{i}^{T}+\pi_{i i} P_{i}\right)^{\star}, \\
\Phi_{i j} & = \begin{cases}\left(\bar{A}_{i}^{T} P_{i}+C_{i}^{T} L_{i}^{T} P_{i}+\pi_{i i} P_{i}\right)^{\star}, & \text { if } i=j \\
\pi_{i j} P_{j}+\pi_{j i} P_{i}, & \text { if } i \neq j .\end{cases}
\end{aligned}
$$

Then, the gain of the designed observer can be computed by

$$
L_{i}=P_{i}^{-1} Y_{i}
$$

Proof. Based on condition (12), it is obtained that

$$
\left(\left[\begin{array}{cccc}
\Omega_{1} & \pi_{21} I_{n} & \cdots & \pi_{N 1} I_{n} \\
\pi_{12} I_{n} & \Omega_{2} & \cdots & \pi_{N 2} I_{n} \\
\vdots & \vdots & \ddots & \vdots \\
\pi_{1 N} I_{n} & \pi_{2 N} I_{n} & \cdots & \Omega_{N}
\end{array}\right] P\right)^{*}<0
$$

where $\Omega_{i}=\bar{A}_{i}^{T}+C_{i}^{T} L_{i}^{T}+\pi_{i i} I_{n}, \forall i \in \mathbb{S}$. In order to make the solvable conditions with LMI forms, we let matrix $P$ selected to be a diagonal form, that is, $P=\operatorname{diag}\left\{P_{1}, P_{2}, \ldots, P_{N}\right\}>0$. Then, (49) is rewritten to be

$$
\left[\begin{array}{cccc}
\Phi_{11} & \Phi_{12} & \cdots & \Phi_{1 N} \\
* & \Phi_{22} & \cdots & \Phi_{2 N} \\
\vdots & \vdots & \ddots & \vdots \\
* & * & \cdots & \Phi_{N N}
\end{array}\right]<0
$$

From (48), it is known that (46) is equal to (50). Because of $P_{i}>0$ and by condition (45), it is known that $-P_{i}$ is Hurwitz and $-P_{i} \in \mathbb{M}_{n}$. Based on [51], one has $-P_{i}^{-1} \preceq 0$ which is equivalent to $P_{i}^{-1} \in \mathbb{R}_{+}^{n \times n}$. Since $Y_{i} \in \mathbb{R}_{+}^{n \times p}$, it is obtained that $L_{i}=P_{i}^{-T} Y_{i} \in \mathbb{R}_{+}^{n \times p}$. Then, $\bar{A}_{i}+L_{i} C_{i} \in \mathbb{M}_{n}, \forall i \in \mathbb{S}$. By Theorem 2, it is known that error system (44) is positive. This completes the proof.

Remark 10. In contrast to some similar results, it is said that the proposed positive observer method has some advantages. On the one hand, the proposed approach is different from the traditional observer design approaches [38, 40-44]. Firstly, in these references, it is seen that there is no positive constraint on the designed observers. In other words, the abovementioned methods are not suitable to positive observer design problem. Secondly, even though such traditional methods are used to design positive observers, it is concluded that the requirement of positivity of observer should be satisfied. However, when the positive constraint must be studied, how to describe it in the obtained results should be considered carefully. Particularly, based on the abovementioned results, it is very difficult to obtain the existence conditions with solvable forms. On the other hand, when the design problem of positive observer is referred to, only a few results are available, in which no jumping and time delay are included. In [46], it is seen that the obtained results were no-convex conditions and cannot be solved easily. Recently, though some LMI conditions for the existence of positive observer were obtained in [47], the related matrices should be diagonal matrices which are more conservative than ones obtained by nondiagonal ones. Based on these observations, it is said that the method proposed here has some advantages in terms of having solvable forms and less conservatism. Moreover, our results are related to systems with Markovian switching and time delay, which are more general.

Remark 11. In this theorem, LMI conditions are provided to compute mode-dependent parameter $L_{i}$. By the methods 
$[9,19]$ dealing with mode-independent problems, it is seen that the proposed theorem can be applied to design modeindependent parameter $L$ similarly, where matrix $P$ will be selected to depend on system mode.

It is said that the design method in Theorem 9 is not easy to be extended to PDMJSs with general TRMs. This is because the TRM in condition (46) has a strong coupling in terms of $\pi_{j i}$ and $\pi_{i j}$ being distributed and in the same position. In order to design observer for PDMJSs with general TRMs, another kind of conditions for constructing observer gain $L_{i}$ based on (16) will be established.

Theorem 12. Consider positive system (1); there exists positive observer (43) such that the resulting error system is asymptotically mean stable, if there are SPVs $\lambda_{i} \in \mathbb{R}_{+}^{n}$ and $\xi_{i} \in \mathbb{R}_{+}^{p}$, $\forall i \in \mathbb{S}$, such that

$$
\lambda_{i}^{T} \bar{A}_{i}+\xi_{i}^{T} C_{i}+\sum_{j=1}^{N} \pi_{i j} \lambda_{j}^{T} \prec 0 .
$$

Then, the corresponding gain of observer (43) is constructed as

$$
L_{i}=\frac{v_{i} \xi_{i}^{T}}{\lambda_{i}^{T} v_{i}}
$$

where $v_{i} \in \mathbb{R}_{+}^{n}$ is any given nonzero vector.

Proof. From condition (16), it is known that

$$
\lambda_{i}^{T}\left(\bar{A}_{i}+L_{i} C_{i}\right)+\sum_{j=1}^{N} \pi_{i j} \lambda_{j}^{T} \prec 0
$$

is a necessary and sufficient condition for the resulting error system asymptotically mean stable. Here, $L_{i} \in \mathbb{R}_{+}^{n \times p}$ should be an SPV such that $\bar{A}_{i}+L_{i} C_{i} \in \mathbb{M}_{n}$. By the representation of $L_{i}$, it is seen that this assumption is obvious. Applying (52) to the original system, we have

$$
\lambda_{i}^{T} \bar{A}_{i}+\lambda_{i}^{T} \frac{v_{i} \xi_{i}^{T}}{\lambda_{i}^{T} v_{i}} C_{i}+\sum_{j=1}^{N} \pi_{i j} \lambda_{j}^{T} \prec 0
$$

which is equivalent to (51). This completes the proof.

Based on Theorem 7 and similar to Theorem 12, we have the following theorem.

Theorem 13. Consider positive system (1) with partially unknown TRM (28); there exists positive observer (43) such that the resulting error system is asymptotically mean stable, if there are SPVs $\lambda_{i} \in \mathbb{R}_{+}^{n}, \xi_{i} \in \mathbb{R}_{+}^{p}$, and $w_{i} \in \mathbb{R}_{+}^{n}, \forall i \in \mathbb{S}$, satisfying (32), $\forall j \neq i \in \overline{\mathbb{S}}_{k}^{i}$ and

$$
\begin{aligned}
& \lambda_{i}^{T} \bar{A}_{i}+\xi_{i}^{T} C_{i}+\sum_{j \neq i, j \in \mathbb{S}_{k}^{i}} \pi_{i j}\left(\lambda_{j}^{T}-\lambda_{i}^{T}-w_{i}^{T}\right)-\pi_{i i} w_{i}^{T} \\
& \quad \prec 0, \quad i \in \mathbb{S}_{k}^{i},
\end{aligned}
$$

$$
\begin{array}{r}
\lambda_{i}^{T} \bar{A}_{i}+\xi_{i}^{T} C_{i}+\sum_{j \neq i, j \in \mathbb{S}_{k}^{i}} \pi_{i j}\left(\lambda_{j}^{T}-\lambda_{i}^{T}-w_{i}^{T}\right)-\sigma w_{i}^{T} \prec 0, \\
i \in \overline{\mathbb{S}}_{k}^{i} .
\end{array}
$$

Then, the parameter $L_{i}$ can also be obtained by (52).

Proof. By the proof of Theorem 12, it is seen that the only difference between them is that the TRM here is partially unknown. After substituting (52) into (16) and similar to (33), one has (53) implied by

$$
\begin{aligned}
& \lambda_{i}^{T} \bar{A}_{i}+\lambda_{i}^{T} \frac{v_{i} \xi_{i}^{T}}{\lambda_{i}^{T} v_{i}} C_{i}+\sum_{j \neq i, j \in \mathbb{S}_{k}^{i}} \pi_{i j}\left(\lambda_{j}^{T}-\lambda_{i}^{T}-w_{i}^{T}\right) \\
& -\pi_{i i} w_{i}^{T} \prec 0, \quad i \in \mathbb{S}_{k}^{i}, \\
& \lambda_{i}^{T} \bar{A}_{i}+\lambda_{i}^{T} \frac{v_{i} \xi_{i}^{T}}{\lambda_{i}^{T} v_{i}} C_{i}+\sum_{j \neq i, j \in \mathbb{S}_{k}^{i}} \pi_{i j}\left(\lambda_{j}^{T}-\lambda_{i}^{T}-w_{i}^{T}\right) \\
& -\sigma w_{i}^{T} \prec 0, \quad i \in \overline{\mathbb{S}}_{k}^{i} .
\end{aligned}
$$

Then, the next is similar to the process of Theorem 7 and is omitted here. This completes the proof.

When TRM is uncertain, we could also have the following result.

Theorem 14. Consider positive system (1) with admissible uncertainty (36); there exists positive observer (43) such that the resulting error system is robustly asymptotically mean stable, if there are SPVs $\lambda_{i} \in \mathbb{R}_{+}^{n}, \xi_{i} \in \mathbb{R}_{+}^{p}$, and $w_{i} \in \mathbb{R}_{+}^{n}$, $\forall i \in \mathbb{S}$, satisfying (38), $\forall j \neq i \in \mathbb{S}$, and

$$
\lambda_{i}^{T} \bar{A}_{i}+\xi_{i}^{T} C_{i}+\sum_{j \neq i} \alpha_{i j}\left(\lambda_{j}^{T}-\lambda_{i}^{T}\right)+2 \sum_{j \neq i} \epsilon_{i j} w_{i}^{T} \prec 0 .
$$

In this case, (52) is used to get the gain of the desired observer.

Proof. This proof can be obtained by the proofs of Theorems 8 and 12 easily. Then, it is omitted here. This completes the proof.

From condition (52) used in Theorems 12 and 14, it is seen that the designed gain of observer (43) is mode-dependent. It means the implementation of observer (43) needs its mode signal available online. It is very ideal in many practical applications. In order to deal with this practical situation and remove the ideal assumption, a mode-independent design method is usually chosen. A direct method for getting modeindependent observer is that the parameters used to compute the gain are selected common for all system modes. That is, $v_{i}$, $\xi_{i}$, and $\lambda_{i}$ are selected as $v, \xi$, and; $\lambda$, respectively. In particular, when $\lambda_{i}=\lambda, \forall i \in \mathbb{S}$, the corresponding conditions presented in such theorems will become independent of TRM. For example, (51) becomes $\lambda^{T} \bar{A}_{i}+\xi^{T} C_{i} \prec 0$. Since there is no constraint on TRM, the corresponding results will be more conservative. It is because any TRM can be satisfactory. This 
is contrary to the facts. In order to solve such problems appropriately, we propose another method to compute the observer gain without missing TRM.

Theorem 15. Consider positive system (1); there exists positive observer (43) such that the resulting error system is asymptotically mean stable, if there are SPVs $\lambda_{i} \in \mathbb{R}_{+}^{n}$ and $\xi \in \mathbb{R}_{+}^{p}$, $\forall i \in \mathbb{S}$, such that

$$
\lambda_{i}^{T} \bar{A}_{i}+\xi^{T} C_{i}+\sum_{j=1}^{N} \pi_{i j} \lambda_{j}^{T} \prec 0 .
$$

Then, the mode-independent gain of observer (43) is constructed as

$$
L=\frac{v \xi^{T}}{\hat{\lambda}^{T} v},
$$

where $v \in \mathbb{R}_{+}^{n}$ is any given nonzero vector and $\hat{\lambda}=\left[\widehat{\lambda}_{i}\right] \in \mathbb{R}_{+}^{n}$ is an SPV whose element is defined as $\hat{\lambda}_{i}=\lambda_{i}^{1} \vee \lambda_{i}^{2} \vee \cdots \vee \lambda_{i}^{N}$ and $\lambda_{i}^{j}$ coming from $\lambda_{j}=\left[\lambda_{i}^{j}\right] \in \mathbb{R}_{+}^{n}, i=\{1,2, \ldots, n\}$. Here, $S P V \lambda_{i}$ is the solution to (58).

Proof. Similar to the proof of Theorem 12, applying (59) to (53), we have

$$
\lambda_{i}^{T} \bar{A}_{i}+\lambda_{i}^{T} \frac{v \xi^{T}}{\hat{\lambda}^{T} v} C_{i}+\sum_{j=1}^{N} \pi_{i j} \lambda_{j}^{T} \prec 0 .
$$

Because of $v \in \mathbb{R}_{+}^{n}$ nonzero and $\lambda_{i}^{T}$ being an SPV, it is concluded that

$$
\hat{\lambda}^{T} v \geq \lambda_{i}^{T} v>0
$$

Based on this and taking into account $C_{i} \in \mathbb{R}_{+}^{p \times n}$ and $\xi \in \mathbb{R}_{+}^{p}$, one gets (60) implied by (58). This completes the proof.

As for the cases of TRM having general forms, by using Theorems 13-14, one could obtain the similar results for mode-independent observer easily. So, the corresponding statements are omitted here.

\section{Numerical Examples}

Example 1. Consider a two-dimensional PDMJS of form (1) with $\eta_{t} \in \mathbb{S}=\{1,2,3\}$, and its parameters are given by

$$
\begin{aligned}
A_{1} & =\left[\begin{array}{cc}
-3 & 1 \\
2 & -3
\end{array}\right], \\
A_{d 1} & =\left[\begin{array}{ll}
0.3 & 0.2 \\
0.1 & 0.4
\end{array}\right], \\
C_{1} & =\left[\begin{array}{cc}
0.1 & 1 \\
0.2 & 0.5
\end{array}\right], \\
A_{2} & =\left[\begin{array}{cc}
-2 & 2 \\
1 & -2
\end{array}\right],
\end{aligned}
$$

$$
\begin{aligned}
A_{d 2} & =\left[\begin{array}{ll}
0.1 & 0.2 \\
0.3 & 0.4
\end{array}\right], \\
C_{2} & =\left[\begin{array}{ll}
0.2 & 2 \\
1 & 1
\end{array}\right], \\
A_{3} & =\left[\begin{array}{ll}
-1 & 0.5 \\
1 & -2.5
\end{array}\right], \\
A_{d 3} & =\left[\begin{array}{ll}
0.3 & 0.1 \\
0.1 & 0.2
\end{array}\right], \\
C_{3} & =\left[\begin{array}{ll}
1 & 0.1 \\
1 & 0.2
\end{array}\right] .
\end{aligned}
$$

Time delay is assumed to be $d=0.2$. First, TRM $\Pi$ is exactly known and given as

$$
\Pi=\left[\begin{array}{ccc}
-1.5 & 0.6 & 0.9 \\
0.7 & -1.2 & 0.5 \\
1.5 & 1.4 & -2.9
\end{array}\right] .
$$

By investigating the results about MJSs, it is said that most of them are generally classified into two classes: modedependent and mode-independent ones. Compared with the mode-independent method designing a common controller or observer for all system modes, mode-dependent method is less conservative. Thus, when the system mode of an MJS is available online, mode-dependent method will be preferable. By Theorem 9, we have the parameters of mode-dependent observers computed as follows:

$$
\begin{aligned}
& L_{1}=\left[\begin{array}{ll}
0.9488 & 1.4628 \\
1.0995 & 1.4370
\end{array}\right], \\
& L_{2}=\left[\begin{array}{ll}
0.2996 & 0.3060 \\
0.2585 & 0.2804
\end{array}\right], \\
& L_{3}=\left[\begin{array}{ll}
1.5224 & 1.3117 \\
1.2311 & 1.2053
\end{array}\right] .
\end{aligned}
$$

Under the initial condition $e_{0}=\left[\begin{array}{ll}1 & 0.5\end{array}\right]^{T}$, we have the simulation of system mode shown in Figure 1, while the response of the resulting error system is demonstrated in Figure 2.

On the other hand, we can also design mode-dependent observer by Theorem 12. Without loss of generality, the corresponding nonzero vector $v_{i}$ used in (4) is given as follows:

$$
\begin{aligned}
& v_{1}=\left[\begin{array}{c}
0.2 \\
1
\end{array}\right], \\
& v_{2}=\left[\begin{array}{l}
2 \\
1
\end{array}\right],
\end{aligned}
$$




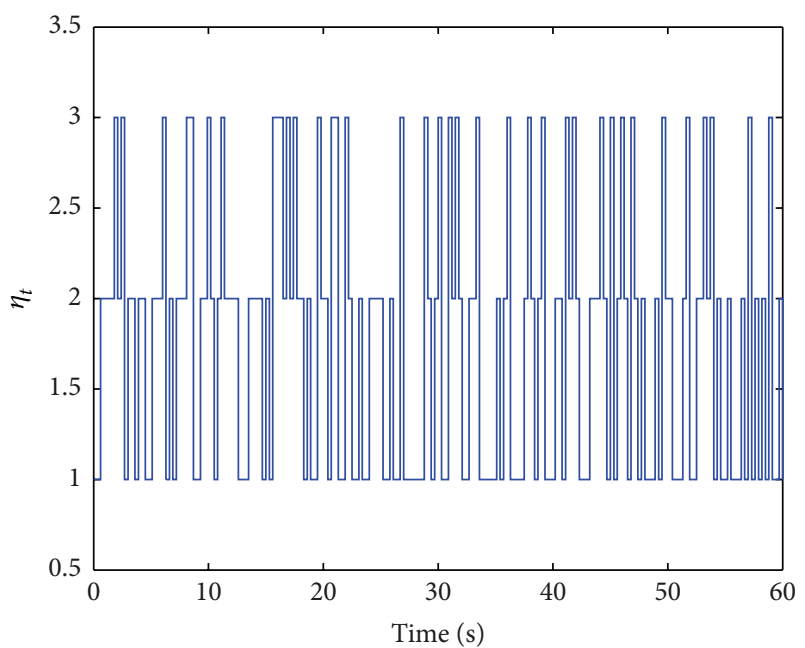

- $\eta_{t}$

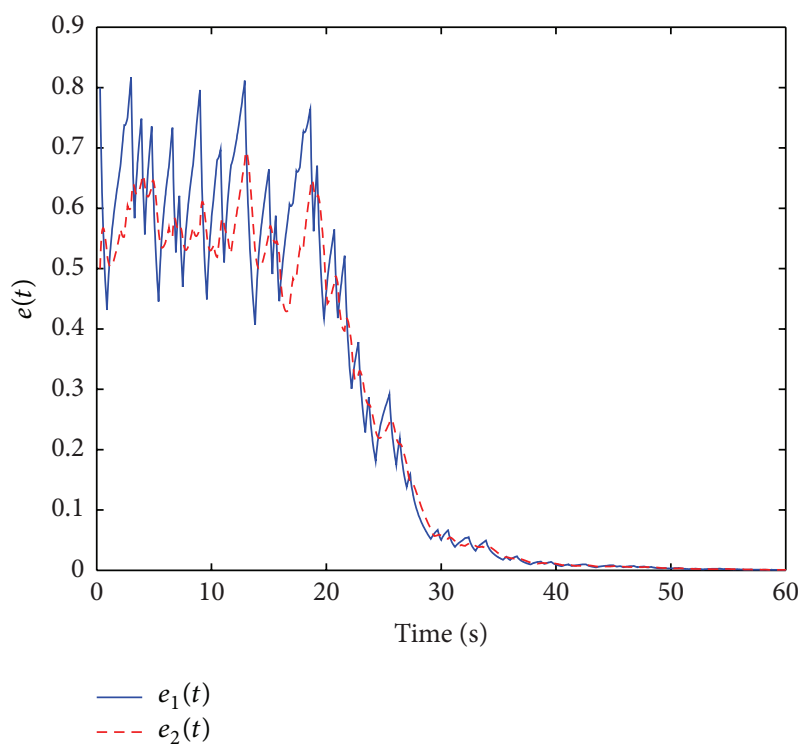

FIGURE 2: The response of error $e(t)$.

$$
v_{3}=\left[\begin{array}{l}
3 \\
1
\end{array}\right] \text {. }
$$

Then, we have the corresponding gains of mode-dependent observer computed as follows:

$$
\begin{aligned}
& L_{1}=\left[\begin{array}{ll}
0.0547 & 0.0732 \\
0.2733 & 0.3662
\end{array}\right], \\
& L_{2}=\left[\begin{array}{ll}
0.0152 & 0.0234 \\
0.0076 & 0.0117
\end{array}\right], \\
& L_{3}=\left[\begin{array}{ll}
0.2242 & 0.2164 \\
0.0747 & 0.0721
\end{array}\right] .
\end{aligned}
$$

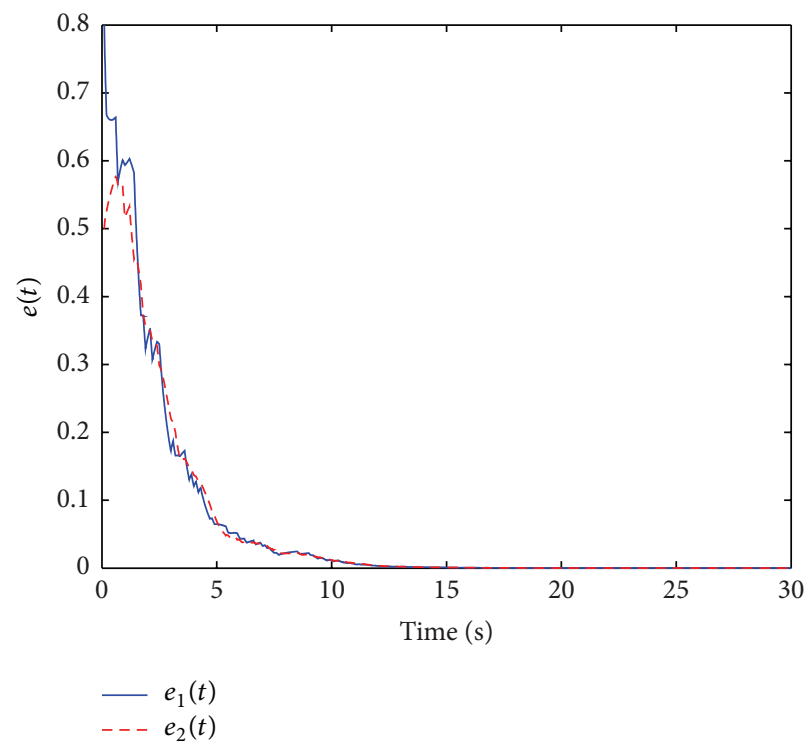

FIgURE 3: The curve of error $e(t)$.

Under the same initial condition, we can have the curve of the resulting error system shown in Figure 3.

When $\Pi$ is partially unknown, it is assumed to be

$$
\Pi=\left[\begin{array}{ccc}
? & 0.6 & ? \\
0.7 & -1.2 & 0.5 \\
? & ? & -2.9
\end{array}\right]
$$

where "?" is denoted as its element unknown or inaccessible, and $\sigma$ is assumed to be $\sigma=-1.5$. With the same given nonzero vector $v_{i}$ and by Theorem 13, the gains of the desired observer can be computed by (52) and presented as follows:

$$
\begin{aligned}
& L_{1}=\left[\begin{array}{ll}
0.0300 & 0.0409 \\
0.1501 & 0.2045
\end{array}\right], \\
& L_{2}=\left[\begin{array}{ll}
0.0185 & 0.0221 \\
0.0092 & 0.0110
\end{array}\right], \\
& L_{3}=\left[\begin{array}{ll}
0.0141 & 0.0137 \\
0.0047 & 0.0046
\end{array}\right] .
\end{aligned}
$$

Under the same initial condition, the state response of error $e(t)$ is simulated in Figure 4.

Finally, if TRM $\Pi$ is an estimation, whose uncertainties are $\left|\Delta \pi_{i j}\right| \leq \epsilon_{i j} \triangleq 0.6 \pi_{i j}, \forall i, j \in \mathbb{S}=\{1,2,3\}$, and $i \neq j$, by 


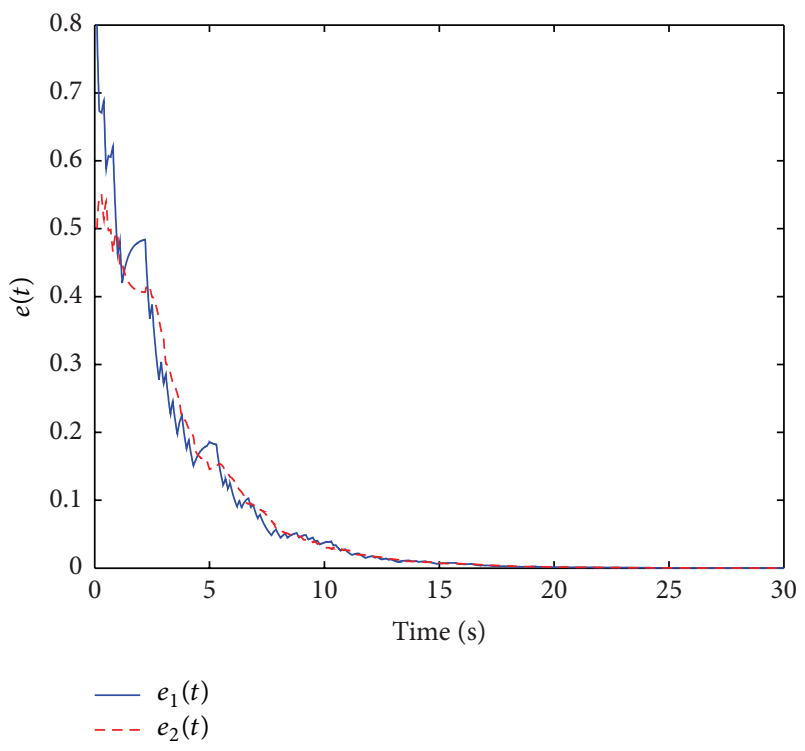

FIgURE 4: The response of error $e(t)$ with partially unknown $\Pi$.

Theorem 14, one can get the corresponding gains of observer listed as follows:

$$
\begin{aligned}
L_{1} & =\left[\begin{array}{ll}
0.0163 & 0.0208 \\
0.0817 & 0.1038
\end{array}\right], \\
L_{2} & =\left[\begin{array}{ll}
0.0093 & 0.0107 \\
0.0047 & 0.0053
\end{array}\right], \\
L_{3} & =\left[\begin{array}{ll}
0.0456 & 0.0446 \\
0.0152 & 0.0149
\end{array}\right] .
\end{aligned}
$$

Under the same condition mentioned above, we have the simulation of error $e(t)$ demonstrated in Figure 5.

When system mode is unavailable, it is said that the designed mode-dependent observers will be disabled. The reason is that the designed mode-dependent observer needs its operation mode available online. However, in some practical cases where the data is transmitted through unreliable networks, it is impossible to obtain all the information online. In other words, because of the effects of networks such as induced delay and packet dropout existing, the transmitted signal will be obtained randomly or with some probability instead of being totally available. In this sense, it is said that sometimes mode-dependent method is very ideal and will limit the scope of application. Thus, it is necessary to propose a method without mode information, where modeindependent method is usually exploited and very suitable to deal with such practical cases. Let the nonzero vector $v$ given as $v=\left[\begin{array}{ll}0.2 & 1\end{array}\right]^{T}$; by Theorem 15, one has the parameter of the mode-independent observer computed as

$$
L=\left[\begin{array}{ll}
0.0082 & 0.0173 \\
0.0412 & 0.0865
\end{array}\right]
$$

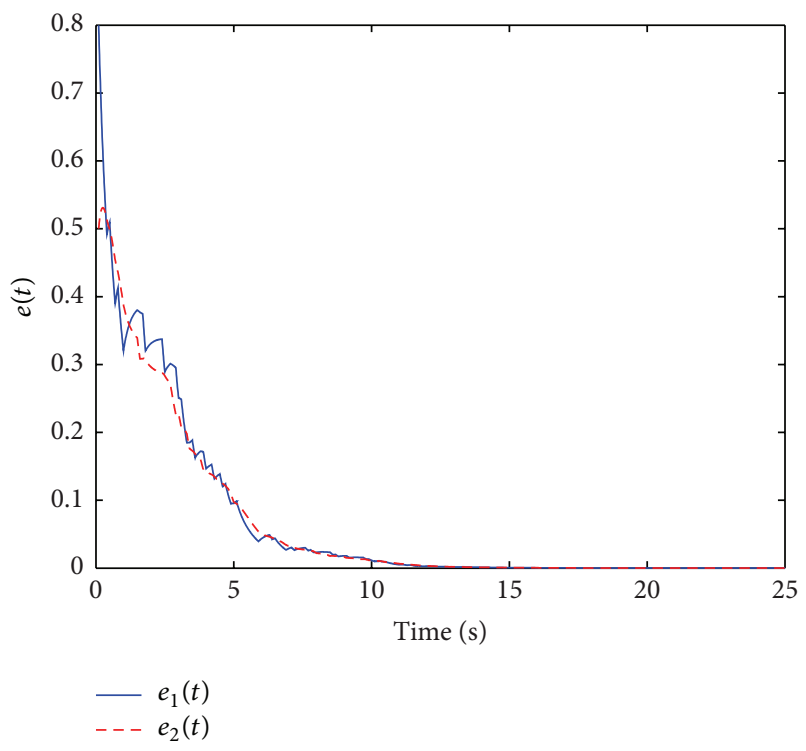

FIGURE 5: The response of error $e(t)$ with uncertain $\Pi$.

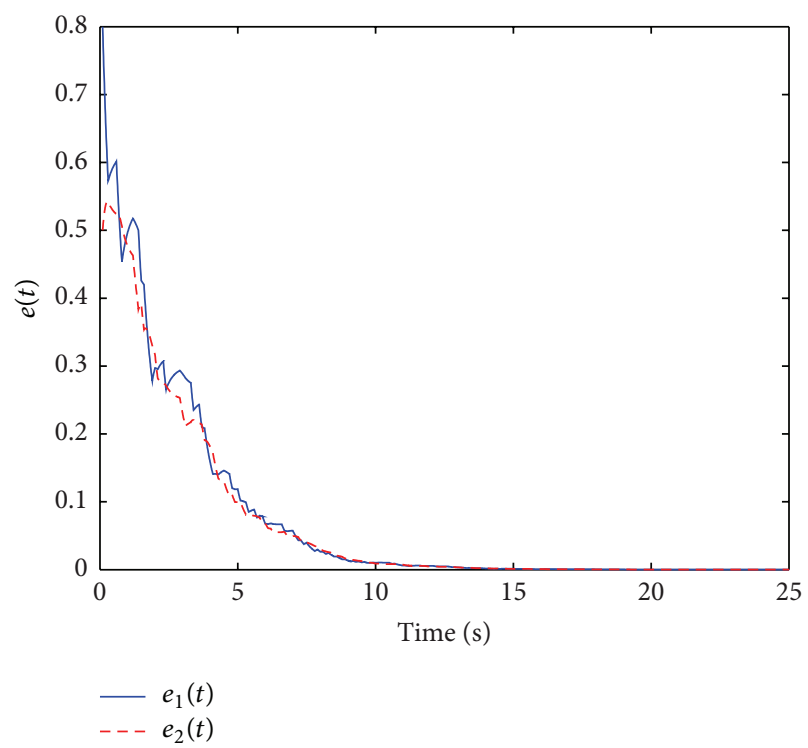

Figure 6: The curve of error $e(t)$ by mode-independent observer.

Applying the designed observer and under the same initial condition, we have the curve of the error system given in Figure 6.

Based on these simulations, it is said that the designed observers are all useful which also demonstrate the effectiveness of the proposed methods.

\section{Conclusions}

In this paper, the positive observer design problem of PDMJSs has been addressed. Necessary and sufficient condition for the positivity of PDMJSs has been developed. Several kinds of equivalent conditions for checking the asymptotically mean stability of DMJSs have been proposed, 
which can be solved easily. It has also showed that the asymptotically mean stability of PDMJSs can be reduced to check the asymptotic stability of a deterministic system without time delay. Based on the presented results, several sufficient conditions for the existence of both mode-dependent and mode-independent observers are developed with solvable forms. All the results have been further extended to some general cases that TRM is partially unknown or uncertain. Particularly, based on the methods proposed in this paper, some extended problems such as filtering for MJSs with asynchronous switching $[52,53]$ could be further considered, which will be our future research topics.

\section{Competing Interests}

Guoliang Wang declares that there are no competing interests regarding the publication of this paper.

\section{Acknowledgments}

This work was supported by the National Natural Science Foundation of China under Grants 61104066, 61203001, 61374043, and 61473140, the China Postdoctoral Science Foundation funded project under Grant 2012M521086, the Program for Liaoning Excellent Talents in University under Grant LJQ2013040, and the Natural Science Foundation of Liaoning Province under Grant 2014020106.

\section{References}

[1] E. K. Boukas, Stochastic Switching Systems: Analysis and Design, Springer, New York, NY, USA, 2005.

[2] E. K. Boukas, "Manufacturing systems: LMI approach," IEEE Transactions on Automatic Control, vol. 51, no. 6, pp. 1014-1018, 2006.

[3] J. Xiong, J. Lam, H. Gao, and D. W. Ho, “On robust stabilization of Markovian jump systems with uncertain switching probabilities," Automatica, vol. 41, no. 5, pp. 897-903, 2005.

[4] L. Li and V. A. Ugrinovskii, "On necessary and sufficient conditions for $H_{\infty}$ output feedback control of Markov jump linear systems," IEEE Transactions on Automatic Control, vol. 52, no. 7, pp. 1287-1292, 2007.

[5] H. P. Liu, D. W. C. Ho, and F. C. Sun, "Design of $H_{\infty}$ filter for Markov jumping linear systems with non-accessible mode information," Automatica, vol. 44, no. 10, pp. 2655-2660, 2008.

[6] L. X. Zhang and E.-K. Boukas, "Stability and stabilization of Markovian jump linear systems with partly unknown transition probabilities," Automatica, vol. 45, no. 2, pp. 463-468, 2009.

[7] G. Wang, Q. Zhang, and V. Sreeram, "Partially mode-dependent $H_{\infty}$ filtering for discrete-time Markovian jump systems with partly unknown transition probabilities," Signal Processing, vol. 90, no. 2, pp. 548-556, 2010.

[8] Y. Zhang, Y. He, M. Wu, and J. Zhang, "Stabilization for Markovian jump systems with partial information on transition probability based on free-connection weighting matrices," Automatica, vol. 47, no. 1, pp. 79-84, 2011.

[9] G. L. Wang and Q. L. Zhang, "Robust $\mathbf{H}_{\mathbf{\infty}}$ control of Markovian jump systems with uncertain switching probabilities," Asian Journal of Control, vol. 14, no. 5, pp. 1407-1410, 2012.
[10] H. Y. Li, H. J. Gao, P. Shi, and X. D. Zhao, "Fault-tolerant control of Markovian jump stochastic systems via the augmented sliding mode observer approach," Automatica, vol. 50, no. 7, pp. 1825-1834, 2014.

[11] G. L. Wang, " $H_{\infty}$ control of singular Markovian jump systems with operation modes disordering in controllers," Neurocomputing, vol. 142, pp. 275-281, 2014.

[12] G. L. Wang, Q. L. Zhang, and X. G. Yan, Analysis and Design of Singular Markovian Jump Systems, Springer, Basel, Switzerland, 2015.

[13] X. R. Mao, "Exponential stability of stochastic delay interval systems with Markovian switching," IEEE Transactions on Automatic Control, vol. 47, no. 10, pp. 1604-1612, 2002.

[14] S. Y. Xu, T. W. Chen, and J. Lam, "Robust $H_{\infty}$ filtering for uncertain Markovian jump systems with mode-dependent time delays," IEEE Transactions on Automatic Control, vol. 48, no. 5, pp. 900-907, 2003.

[15] S. Xu, J. Lam, and X. Mao, "Delay-dependent $H_{\infty}$ control and filtering for uncertain Markovian jump systems with timevarying delays," IEEE Transactions on Circuits and Systems. I. Regular Papers, vol. 54, no. 9, pp. 2070-2077, 2007.

[16] H. Y. Li, B. Chen, Q. Zhou, and W. Y. Qian, "Robust stability for uncertain delayed fuzzy Hopfield neural networks with Markovian jumping parameters," IEEE Transactions on Systems, Man, and Cybernetics Part B: Cybernetics, vol. 39, no. 1, pp. 94102, 2009.

[17] L. Hu, X. Mao, and L. Zhang, "Robust stability and boundedness of nonlinear hybrid stochastic differential delay equations," IEEE Transactions on Automatic Control, vol. 58, no. 9, pp. 23192332, 2013.

[18] C. Y. Han, H. S. Zhang, and M. Y. Fu, "Optimal filtering for networked systems with Markovian communication delays," Automatica, vol. 49, no. 10, pp. 3097-3104, 2013.

[19] G. L. Wang and S. Y. Xu, "Robust $H_{\infty}$ filtering for singular time-delayed systems with uncertain Markovian switching probabilities," International Journal of Robust and Nonlinear Control, vol. 25, no. 3, pp. 376-393, 2015.

[20] L. Farina and S. Rinaldi, Positive Linear Systems: Theory and Applications, Wiley-Interscience, New York, NY, USA, 2000.

[21] B. Cantó, C. Coll, and E. Sánchez, "Positive solutions of a discrete-time descriptor system," International Journal of Systems Science, vol. 39, no. 1, pp. 81-88, 2008.

[22] E. Klipp, R. Herwig, A. Kowald, C. Wierlig, and H. Lehrach, Systems Biology in Practice: Concepts, Implementation and Application, John Wiley \& Sons, Berlin, Germany, 2005.

[23] R. Shorten, F. Wirth, and D. Leith, "A positive systems model of TCP-like congestion control: asymptotic results," IEEE/ACM Transactions on Networking, vol. 14, no. 3, pp. 616-629, 2006.

[24] L. Benvenuti and L. Farina, "A tutorial on the positive realization problem," IEEE Transactions on Automatic Control, vol. 49, no. 5, pp. 651-664, 2004.

[25] C. Commault and M. Alamir, "On the reachability in any fixed time for positive continuous-time linear systems," Systems and Control Letters, vol. 56, no. 4, pp. 272-276, 2007.

[26] M. E. Valcher, "On the reachability properties of continuoustime positive systems," in Proceedings of the 16th Mediterranean Conference on Control Automation, pp. 990-995, Ajaccio, France, June 2008.

[27] P. D. Leenheer and D. Aeyels, "Stabilization of positive linear systems," Systems \& Control Letters, vol. 44, no. 4, pp. 259-271, 2001. 
[28] M. A. Rami and F. Tadeo, "Controller synthesis for positive linear systems with bounded controls," IEEE Transactions on Circuits and Systems II: Express Briefs, vol. 54, no. 2, pp. 151-155, 2007.

[29] J.-E. Feng, J. Lam, P. Li, and Z. Shu, "Decay rate constrained stabilization of positive systems using static output feedback," International Journal of Robust and Nonlinear Control, vol. 21, no. 1, pp. 44-54, 2011.

[30] J. F. Zhang, Z. Z. Han, F. B. Zhu, and J. Huang, "Feedback control for switched positive linear systems," IET Control Theory \& Applications, vol. 7, no. 3, pp. 464-469, 2013.

[31] T. Kaczorek, "Realization problem for a class of positive continuous-time systems with delays," International Journal of Applied Mathematics and Computer Science, vol. 15, no. 4, pp. 447-453, 2005.

[32] T. Kaczorek, "Stability of positive continuous-time linear systems with delays," Bulletin of the Polish Academy of Sciences: Technical Sciences, vol. 57, no. 4, pp. 395-398, 2009.

[33] X. Liu, W. Yu, and L. Wang, "Stability analysis for continuoustime positive systems with time-varying delays," IEEE Transactions on Automatic Control, vol. 55, no. 4, pp. 1024-1028, 2010.

[34] Y. M. Zhang, Q. L. Zhang, T. Tanaka, and X.-G. Yan, "Positivity of continuous-time descriptor systems with time delays," IEEE Transactions on Automatic Control, vol. 59, no. 11, pp. 30933097, 2014.

[35] P. Bolzern, P. Colaneri, and G. De Nicolao, "Stochastic stability of positive Markov jump linear systems," Automatica, vol. 50, no. 4, pp. 1181-1187, 2014.

[36] S. Q. Zhu, Q.-L. Han, and C. H. Zhang, " $l_{1}$-gain performance analysis and positive filter design for positive discrete-time Markov jump linear systems: a linear programming approach," Automatica, vol. 50, no. 8, pp. 2098-2107, 2014.

[37] A. M. Pertew, H. J. Marquez, and Q. Zhao, " $H_{\infty}$ observer design for Lipschitz nonlinear systems," IEEE Transactions on Automatic Control, vol. 51, no. 7, pp. 1211-1216, 2006.

[38] S. Sundaram and C. N. Hadjicostis, "Delayed observers for linear systems with unknown inputs," IEEE Transactions on Automatic Control, vol. 52, no. 2, pp. 334-339, 2007.

[39] H. S. Wu, "Adaptive robust state observers for a class of uncertain nonlinear dynamical systems with delayed state perturbations," IEEE Transactions on Automatic Control, vol. 54, no. 6, pp. 1407-1412, 2009.

[40] L. Xie and P. P. Khargonekar, "Lyapunov-based adaptive state estimation for a class of nonlinear stochastic systems," Automatica, vol. 48, no. 7, pp. 1423-1431, 2012.

[41] J. Raouf and E. K. Boukas, "Observer-based controller design for linear singular systems with Markovian switching," in Proceedings of the 43rd IEEE Conference on Decision and Control (CDC '04), pp. 3619-3624, December 2004.

[42] X. Zhang, G. Lu, and Y. Zheng, "Observer design for descriptor Markovian jumping systems with nonlinear perturbations," Circuits, Systems, and Signal Processing, vol. 27, no. 1, pp. 95-112, 2008.

[43] H. L. Dong, Z. D. Wang, and H. J. Gao, “Observer-based $\mathbf{H}_{\mathbf{\infty}}$ control for systems with repeated scalar nonlinearities and multiple packet losses," International Journal of Robust and Nonlinear Control, vol. 20, no. 12, pp. 1363-1378, 2010.

[44] G. Wang and Q. Zhang, "Adaptive state estimation for stochastic delay systems with state-dependent Markovian switching," IET Control Theory \& Applications, vol. 6, no. 6, pp. 822-828, 2012.
[45] J. Back and A. Astolfi, "Existence conditions and a constructive design of positive linear observers for positive linear systems," in Proceedings of the 45th IEEE Conference on Decision and Control (CDC '06), pp. 4734-4739, San Diego, Calif, USA, December 2006.

[46] H. M. Hardin and J. H. van Schuppen, "Observers for linear positive systems," Linear Algebra and Its Applications, vol. 425, no. 2-3, pp. 571-607, 2007.

[47] Z. Shu, J. Lam, H. Gao, B. Du, and L. Wu, "Positive observers and dynamic output-feedback controllers for interval positive linear systems," IEEE Transactions on Circuits and Systems. I. Regular Papers, vol. 55, no. 10, pp. 3209-3222, 2008.

[48] B. Brain, J. Wang, and Z. H. Qu, "Nonlinear positive observer deisng for positive dynamical systems," in Proceedings of the In American Control Conference (ACC '10), pp. 6231-6237, Baltimore, Md, USA, June-July 2010.

[49] S. P. He and F. Liu, "Finite-time $H_{\infty}$ fuzzy control of nonlinear jump systems with time delays via dynamic observer-based state feedback," IEEE Transactions on Fuzzy Systems, vol. 20, no. 4, pp. 605-614, 2012.

[50] X. R. Mao and C. G. Yuan, Stability of Stochastic Differential Equations with Markovian Switching, Imperial College Press, London, UK, 2006.

[51] O. Mason and R. Shorten, "On linear copositive Lyapunov functions and the stability of switched positive linear systems," IEEE Transactions on Automatic Control, vol. 52, no. 7, pp. 13461349, 2007.

[52] L. Zhang, Y. Zhu, and W. X. Zheng, "Energy-to-peak state estimation for Markov jump RNNs with time-varying delays via nonsynchronous filter with nonstationary mode transitions," IEEE Transactions on Neural Networks and Learning Systems, vol. 26, no. 10, pp. 2346-2356, 2015.

[53] L. X. Zhang, Y. Z. Zhu, P. Shi, and Y. X. Zhao, "Resilient asynchronous $H_{\infty}$ filtering for Markov jump neural networks with unideal measurements and multiplicative noises," IEEE Transactions on Cybernetics, vol. 45, no. 12, pp. 2840-2852, 2015. 


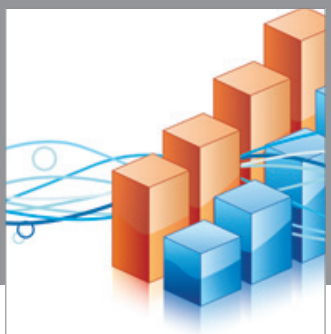

Advances in

Operations Research

vatem alat4

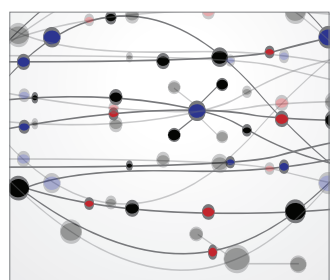

\section{The Scientific} World Journal
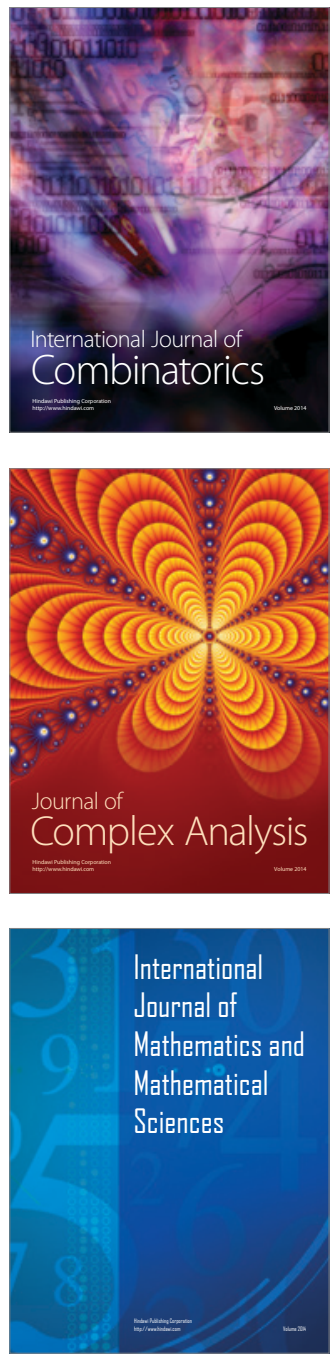
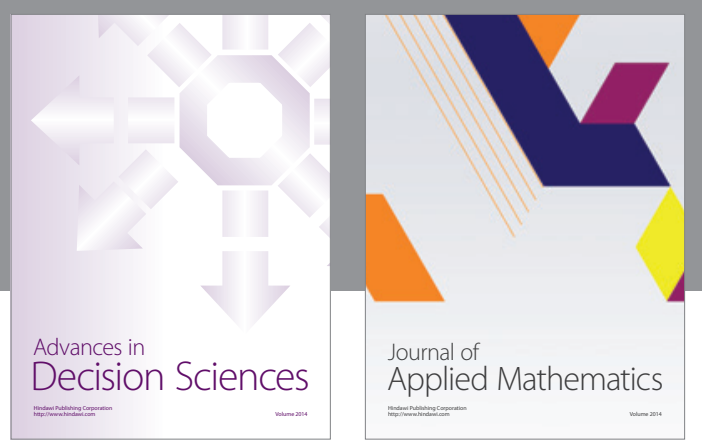

Algebra

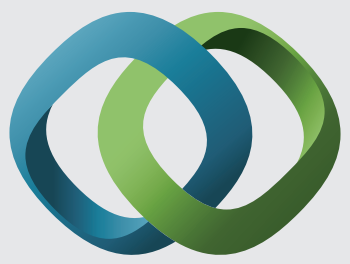

\section{Hindawi}

Submit your manuscripts at

http://www.hindawi.com
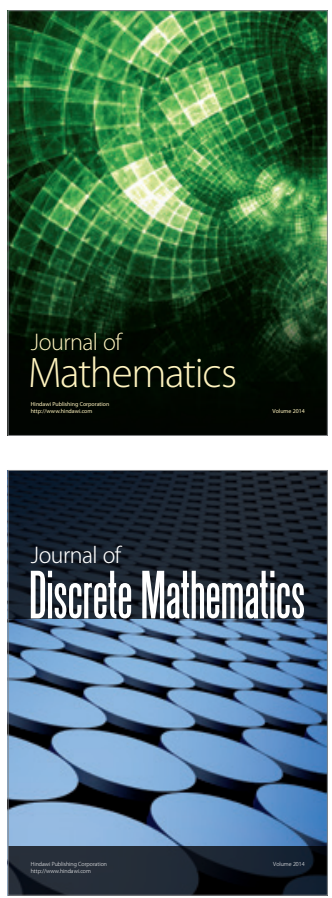

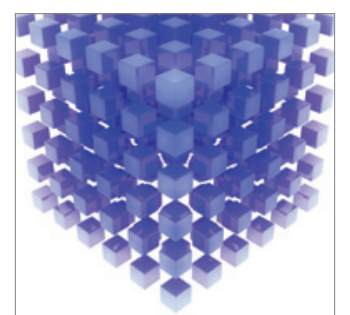

Mathematical Problems in Engineering
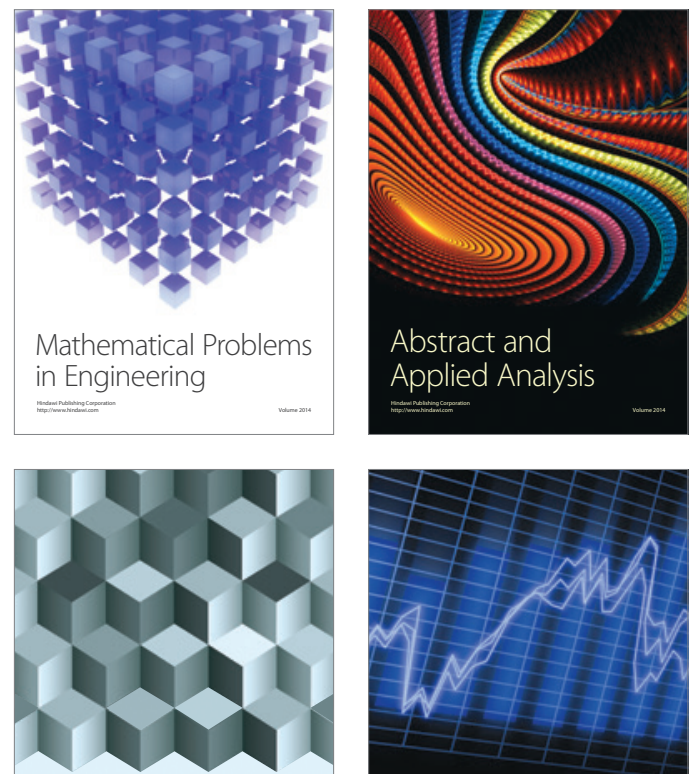

Journal of

Function Spaces

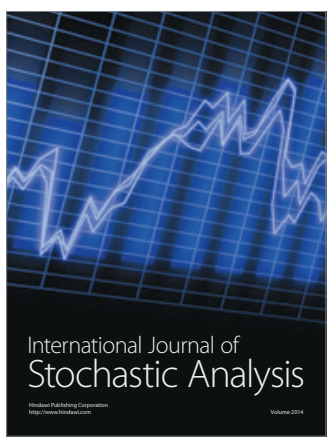

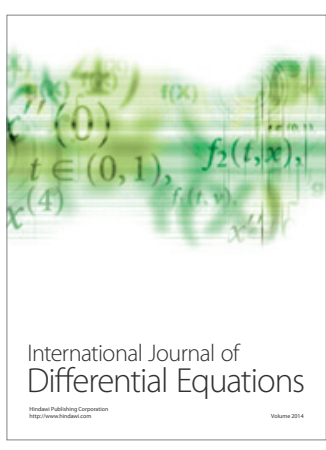
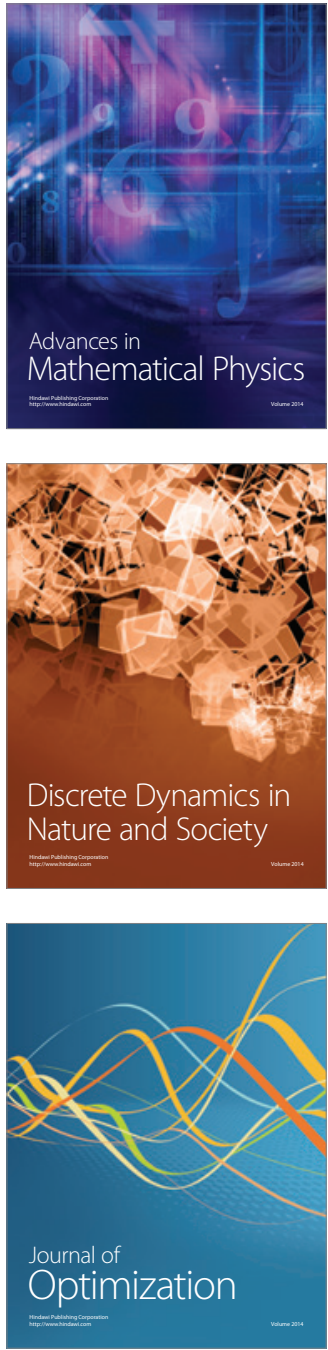